\title{
A Micromachined Vibration Isolation System for a 1 GHz STW Resonator
}

\author{
J. Robert Reid", Member IEEE, Victor M. Bright ${ }^{*}$, Member IEEE, John A. Kosinski ${ }^{+}$, Senior Member IEEE \\ "Rome Laboratory, Hanscom AFB, MA \\ *Air Force Institute of Technology, Wright Patterson AFB, OH \\ ${ }^{+}$U.S. Army Communications-Electronics Command, Fort Monmouth, NJ
}

\begin{abstract}
$\underline{\text { Abstract }}$
A micromachined system has been developed for reducing the vibration sensitivity of surface transverse wave (STW) resonators. The isolation system consists of a support platform for mounting the STW resonator, four support arms, and a support rim. The entire isolation system measures $8 \mathrm{~mm}$ by $9 \mathrm{~mm}$ by $0.4 \mathrm{~mm}$ without the resonator mounted on the platform. The system acts as a passive vibration isolation system, decreasing the magnitude of high frequency ( $>1.2 \mathrm{kHz}$ ) vibrations. Vibration sensitivity measurements were taken for vibrations with frequencies ranging from $100 \mathrm{~Hz}$ to $5 \mathrm{kHz}$. The measured data show that the system performs as expected with a low frequency $\left(<500 \mathrm{~Hz}\right.$ ) vibration sensitivity of $1.8 * 10^{-8} / \mathrm{g}$ and a high frequency roll off of $12 \mathrm{~dB} /$ octave.
\end{abstract}

\section{$\underline{\text { I. Introduction }}$}

Fixed frequency and voltage controlled oscillators are required for the design of wireless systems such as Doppler radar and satellite based navigation and communication systems [1]. The phase noise of the oscillator has a direct influence on the performance of the system. In particular, oscillator phase noise can lead to increased error rates in digital communication systems and positional inaccuracies in navigation systems, while in Doppler radar systems, oscillator phase noise limits the ability of the radar to detect slow moving targets.

Environmental vibration is a major source of phase noise in crystal based oscillators. Vibration of an oscillator at a specific frequency results in vibration induced sidebands as shown in Fig. 1, while random vibration covering a range of frequencies results in a degradation of the oscillator phase noise close to the carrier [2]. Vibration of the oscillator results in a time varying acceleration being applied to the oscillator. The oscillator is then considered to have an "acceleration sensitivity" which is represented as the vector quantity, $\Gamma$. Under acceleration, the oscillator frequency shifts by an amount which can be calculated as [2]

$\delta f=f_{0}(\boldsymbol{\Gamma} \cdot \mathbf{a})$ where $\delta f$ is the frequency shift of the oscillator, $f_{0}$ is the center frequency of the oscillator, and $\mathbf{a}$ is the applied acceleration vector. The effects of sinusoidal or random vibrations on the oscillator phase noise are readily calculated using Eq. (1) in conjunction with modulation theory [2].

The vibration sensitivity of crystal oscillators has been studied for several decades, but only recently has a good understanding of the problem been acquired [3-5]. The oscillator consists of two systems; the electronic circuit including a low noise amplifier, a tuning network, and a matching network; and the crystal resonator which is often a bulk acoustic wave (BAW) resonator, surface acoustic wave (SAW) resonator, or a surface transverse wave (STW) resonator. Vibration of the oscillator causes a time varying acceleration of both the oscillator circuit, and the resonator. For the oscillator circuit, the vibration results in wire leads shifting and changes the performance of individual components such as air core inductors and dashpots [4]. However, by using rigid printed circuit boards, solid state components such as MMIC amplifiers, and careful circuit design and construction, the effect of vibration on the oscillator circuit can be minimized $[4,6]$. This leaves the crystal resonator as the primary source of

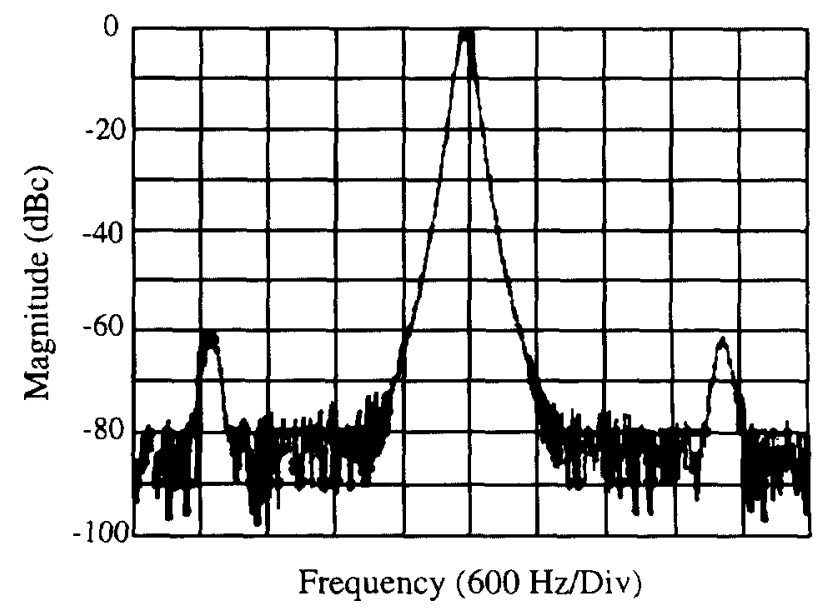

Figure 1. Vibration of a fixed frequency oscillator results in sidebands in the output frequency spectrum. The oscillator shown here has a $1.00 \mathrm{GHz}$ center frequency. A $1 \mathrm{~g}$ vibration at $2250 \mathrm{~Hz}$ is being applied to the oscillator. 
vibration induced phase noise in the oscillator.

Acceleration of the resonator results in body forces being applied to the resonator by the mounting structure which results in a deformation of the crystal [4,7]. This deformation causes a change in the resonator transimpedance $[4,5]$. It has been shown that the vibration sensitivity of the oscillator is dependent not on the shift in the resonator center frequency, but instead on the change in the resonator transimpedance [4,5]. A shift in the resonator transimpedance requires that the operating point of the electronic circuit shift so that the net phase change through the oscillator loop remains zero, and thus the output frequency of the oscillator shifts. Reducing the effect of vibration on the oscillator can then be achieved by one of two methods [4]:

1) Decrease the sensitivity of the resonator transimpedance to the crystal deformation.

2) Minimize or eliminate the vibration.

Micromachining allows the precise fabrication of millimeter to centimeter scale systems with individual components having dimensions of a few micrometers. This research evaluates the use of micromachined systems for reducing the vibration sensitivity of acoustic wave resonators. A micromachined system has been designed around a $1 \mathrm{GHz}$ STW resonator. The system was designed to provide passive vibration isolation to the STW resonator.

\section{Design and Theory}

The primary goal of designing a micromachined isolation system is to provide vibration isolation for the resonator. The basic design of the silicon isolation system is seen in Fig. 2. The system consists of a $4.5 \mathrm{~mm}$ long, $3 \mathrm{~mm}$ wide, $0.4 \mathrm{~mm}$ thick platform for the resonator supported by four $2.0 \mathrm{~mm}$ long, $0.1 \mathrm{~mm}$ wide, $30 \mu \mathrm{m}$ thick arms connected to a $1 \mathrm{~mm}$ wide, $0.4 \mathrm{~mm}$ thick support rim. The system is designed around a $3.86 \mathrm{~mm}$ long, $1.86 \mathrm{~mm}$ wide, $0.5 \mathrm{~mm}$ high $1 \mathrm{GHz}$ STW resonator $[8,9]$. The platform and the

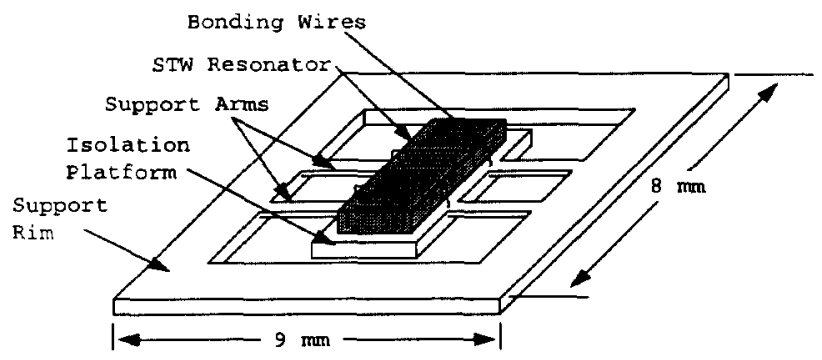

Figure 2. Illustration of the vibration isolation system. The illustration is not to scale. resonator act as a mass supported by the four arms which act as springs. When the base connected to the support rim is vibrated, the vibration is transmitted via the arms to the support platform. As the frequency of the vibration increases above the mechanical resonance frequency of the mass-spring system, the magnitude of the platform vibration is reduced below that of the base. The rigidity of the platform, the location of the support arms, and the mounting of the resonator on the platform determine the shape of the body forces applied to the resonator.

In order to reduce the resonant frequency of the system while maintaining a rigid support, it is necessary to fabricate the support arms thinner than the support platform [10]. Therefore, a fabrication process allowing two silicon thickness levels was developed [11]. The process uses a two sided anisotropic etch of the (110)-cut silicon wafer. The initial etch thins the region of the support arms, while the second etch patterns the top of the arms, the platform, and the support rim.

\section{II.1 Calculation of the Acceleration Sensitivity}

The calculation of the acceleration sensitivity of an isolated STW resonator is similar to the analysis of SAW resonators $[12,13]$. The process begins by calculating the STW mode shape for the resonator. For this research, the resonator is not varied, and therefore the mode shape is only calculated once. After the STW mode shape has been calculated, a finite element model of the resonator and the platform is created. A $1 \mathrm{~g}$ acceleration is applied to the model in the direction normal to the surface of the resonator. Finite element analysis is performed to compute the nodal displacements. The calculated deformations provide the solution to the static biasing problem, allowing the calculation of the spatially varying effective elastic constants. Using Tiersten's perturbation integral [14] in conjunction with the spatially varying effective elastic constants and the STW mode shape, the change in the resonator frequency is calculated, and finally, the acceleration sensitivity of the resonator is calculated.

Finite element analysis (FEA) allowed a numerical study of the effect of several platform parameters on acceleration sensitivity. The following simplifying assumptions were made for the FEA studies:

1) The resonator is rigidly mounted to the platform.

2) The platform and the resonator are the same length.

3) The support arms and the support platform are the same thickness. 
These assumptions affect the absolute calculation of the acceleration sensitivity, however, they do not prevent an analysis of the relative changes in acceleration sensitivity given the changing design parameters.

The finite element analysis shows that increasing the rigidity of platform/resonator combination results in decreased acceleration sensitivity. The analysis also shows that increasing the mass of the platform without a corresponding increase in rigidity results in increased acceleration sensitivity. Finally, it is shown that moving the support arms towards the center of the platform results in decreased acceleration sensitivity. This is caused by a decreased deformation in the active region of the STW resonator. The primary result of the finite element analysis, however, is that modification of the platform dimensions can reduce but not eliminate the acceleration sensitivity. Therefore, vibration isolation becomes the primary method for further reduction of the acceleration sensitivity.

\section{II.2 Calculation of the Vibration Sensitivity}

Calculation of the transmissibility of a vibration from the support rim to the platform is done by modeling the system as a damped mass-spring system as shown in Fig. 3. The mass of the system is computed from the mass of the support platform, and the mass of the resonator. The spring constant, $k_{s y s}$, is computed as

$$
k_{\text {sys }}=4 \cdot\left(k_{1}+k_{2}+k_{3}\right)
$$

where $k_{1}$ is the spring constant due to bending of the beam, $k_{2}$ is the spring constant due to residual stress, and $k_{3}$ is the spring constant due to non-linear forces resulting from large deflections. For the designed system, small

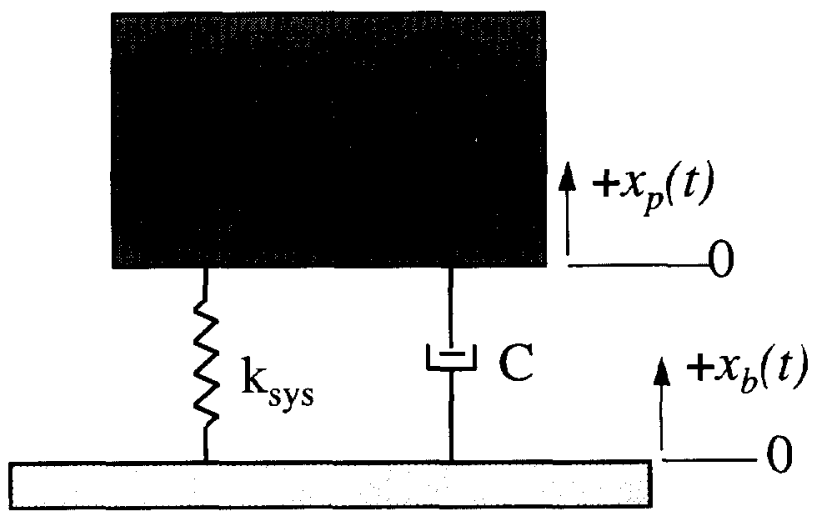

Figure 3. The isolation system is modeled as a damped mass-spring system. The zero position and direction of positive displacement are specified for both the platform, $x_{p}$, and the base, $x_{b}$. deflections can be assumed. Further, the residual stress in undoped (110) silicon is very low. Therefore, $k_{2}$ and $k_{3}$ are neglected and the spring constant is calculated as

$k_{\text {sys }}=4 \cdot k_{1}=4 \cdot \frac{E_{S i} s_{w} t_{w}^{3}}{s_{l}^{3}}$

where $E_{s i}$ is the Young's modulus of silicon, $s_{w}$ is the width of the support beam, $t_{w}$ is the thickness of the support beam and $s_{l}$ is the length of the support beam. The damping coefficient, $C$, is calculated as [15]

$C=\frac{0.42 \cdot A^{2} \cdot \mu}{d^{3}}$

where $A$ is the area of the bottom of the support platform, $\mu$ is the viscosity of air at 1 atmosphere, and $d$ is the separation between the support platform and the oscillator substrate. Table 1 provides a list of the system parameters.

The motion of the system is described as

$$
M_{0} \ddot{x}_{p}+C \dot{x}_{p}-k_{s y s}\left(x_{b}-x_{p}\right)=0
$$

where $\ddot{x}_{p}, \dot{x}_{p}, x_{p}$ are acceleration, velocity, and position of the platform, and $x_{b}$ is the position of the base. The transmissibility, $T_{a}$, of a vibration from the base to the platform is then

$T_{a}=\frac{x_{p}}{x_{b}}$

Solving Eq. (5) for a harmonic motion of the platform results in

$\left|T_{a}\right|=\left[\left(\left(1-\left(\omega / \omega_{0}\right)^{2}\right)^{2}+(C \omega / k)^{2}\right]^{-1 / 2}\right.$

where $\omega=2 \pi f$ is the angular frequency, $f$ is the frequency

Table 1. Calculated System Parameters.

\begin{tabular}{|c|c|}
\hline Parameter & Calculated Value \\
\hline $\mathrm{M}_{0}$ & $2.24 * 10^{-5} \mathrm{~kg}$ \\
\hline $\mathrm{k}_{\mathrm{sys}}$ & $1079.6 \mathrm{~N} / \mathrm{m}$ \\
\hline $\mathrm{f}_{0}$ & $1159.8 \mathrm{~Hz}$ \\
\hline $\mathrm{C}(\mathrm{d}=25 \mu \mathrm{m})$ & $6.18 * 10^{-2} \mathrm{~kg} * \mathrm{~s}$ \\
\hline
\end{tabular}




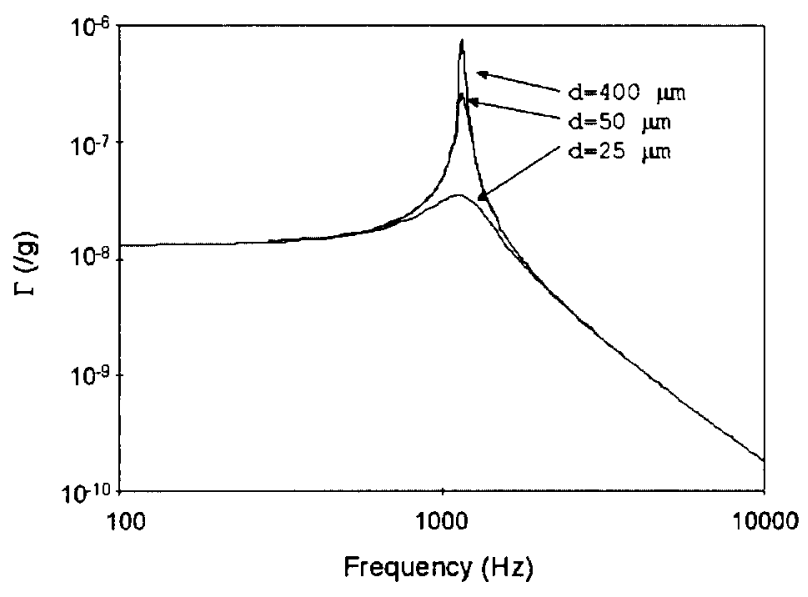

Figure 4. Predicted vibration sensitivity of the isolated STW resonators.

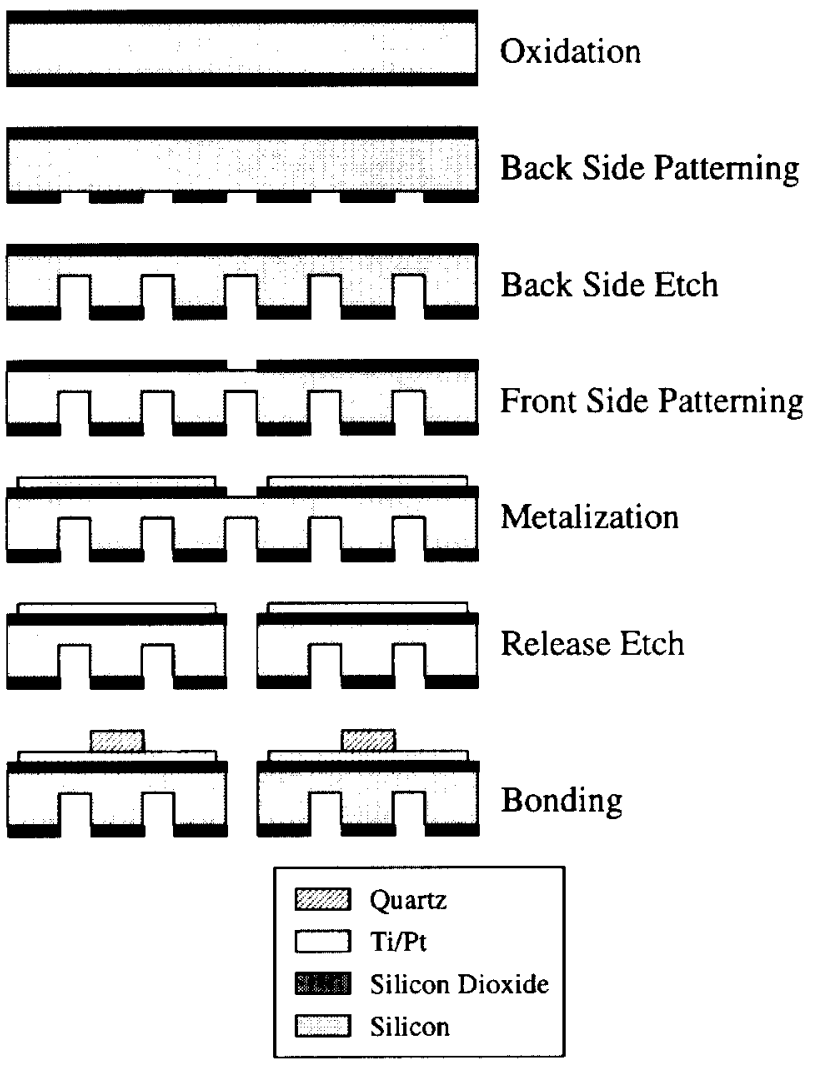

Figure 5. Fabrication process for the silicon isolation systems.

in $\mathrm{Hz}$, and $\omega_{0}=2 \pi f_{0}=\sqrt{k_{\text {sys }} / M_{0}}$ is the angular resonant frequency of the mass-spring system with $f_{0}$ the resonant frequency in $\mathrm{Hz}$.
The predicted vibration sensitivity of the system is the product of the transmissibility and the predicted acceleration sensitivity. Using a value of $1.3 * 10^{-8} / \mathrm{g}$ from the finite element analysis, and the values calculated for $k_{\text {sys }}, f_{0}, C$, and $M_{0}$ (see Table 1 ), the vibration sensitivity of the isolated resonator can be calculated. The predicted vibration sensitivity is shown in Fig. 4.

\section{Fabrication}

The basic fabrication process is shown in Fig. 5. The process is described in detail in reference [11] Fabrication begins with commercially available, 3 inch diameter, $0.4 \mathrm{~mm}$ thick, double side polished, (110)-cut silicon wafers with the primary wafer flat aligned to the (111) crystal plane. Several (111) planes are normal to the (110) wafer surface, and it is therefore possible to etch deep vertical structures into the surface of the wafer using a highly selective anisotropic etchant such as potassium hydroxide $(\mathrm{KOH})[16,17]$ or tetramethyl ammonium hydroxide (TMAH) [18]. KOH was chosen for this work due to its high etch selectivity for both (111):(110) crystal planes $(>400)$ and $(110): \mathrm{SiO}_{2}(>200)[16,17]$. Oxide is the preferred masking material due to the availability of both thermal oxidation furnaces and chemical etchants. A $2.4 \mu \mathrm{m}$ thick oxide is thermally grown at $1100^{\circ} \mathrm{C}$ with steam flowing through the furnace at a rate of 1 liter per minute. Patterning is done using a standard photolithography process. The oxide is etched in a solution of hydrofluoric acid (HF) buffered in ammonium fluoride $\left(\mathrm{NH}_{4} \mathrm{~F}: \mathrm{HF} 4: 1\right)$. Silicon etches are performed with a solution of $\mathrm{KOH}$ in deionized water ( $50 \%$ by wt.). The solution is placed in a reflux container and raised to $85^{\circ} \mathrm{C}$. The $(110)$ silicon etch rate was measured at 2.1 $\mu \mathrm{m} / \mathrm{min}$. By utilizing only commonly available equipment and common processing techniques, the fabrication process provides a simple and inexpensive method to produce vibration isolation platforms.

$1 \mathrm{GHz}$ STW resonators provided by SAWTEK, Inc. [8,9] were mounted on the center of the isolation platforms using Ablebond 84-1LMI conductive epoxy. The epoxy was cured at $160^{\circ} \mathrm{C}$ for 30 minutes. The resonators were then mounted on hybrid oscillators also provided by SAWTEK, Inc. [8,9]. Figure 6 shows a diagram of the resonator mounting. Ablebond 84-3LMI non-conductive epoxy is deposited in the area underneath the isolator support rim. The isolators are then pushed onto the epoxy. The cured epoxy has a thickness of 20 to $30 \mu \mathrm{m}$. That spacing serves as the spacing between the support platform and the oscillator circuit board. Figure 7 shows a photograph of the isolation system with a $1 \mathrm{GHz}$ STW resonator mounted on the support platform. 


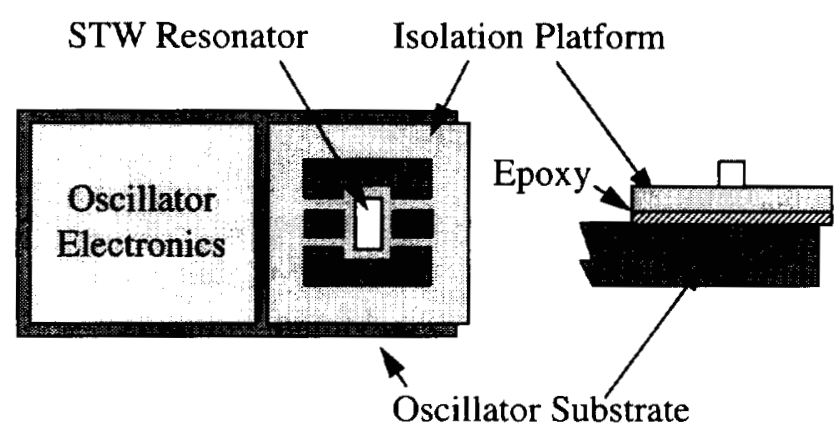

Figure 6. The isolation platform is mounted with epoxy under the support rim only. This leaves a 20-30 $\mu \mathrm{m}$ spacing between the support platform and the hybrid oscillator substrate. The size of the isolation system required that it over hang the edge of the oscillator substrate by approximately $0.5 \mathrm{~mm}$ to $1.0 \mathrm{~mm}$. Epoxy was used to ensure a rigid mount under the overhanging edge.

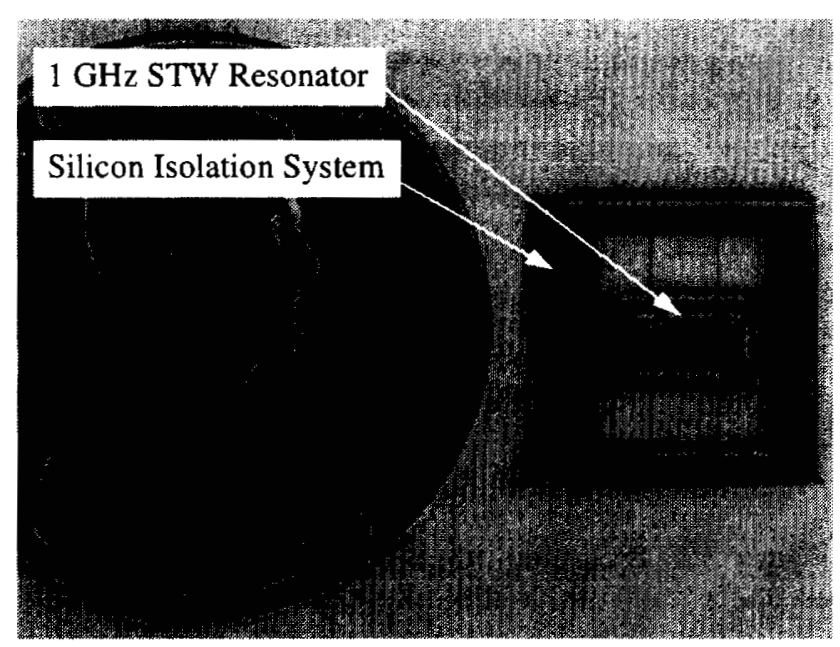

Figure 7. Photograph of a $1 \mathrm{GHz}$ STW resonator mounted on the silicon isolation system. A US penny is used for size reference.

The resonators were bonded directly from the STW resonator to the oscillator bonding pads using an ultrasonic ball bonder with $25 \mu \mathrm{m}$ diameter gold wire. Note that the effect of the bond wires on the system resonant frequency was ignored due to the relatively low Young's modulus of gold compared to silicon, the length of the bonding wires, and the small cross section of the wires.

\section{Experimental Results}

Isolated resonators were mounted on the four available hybrid oscillators. Figure 8 shows the open loop gain and phase for one of the oscillators. At the operating point

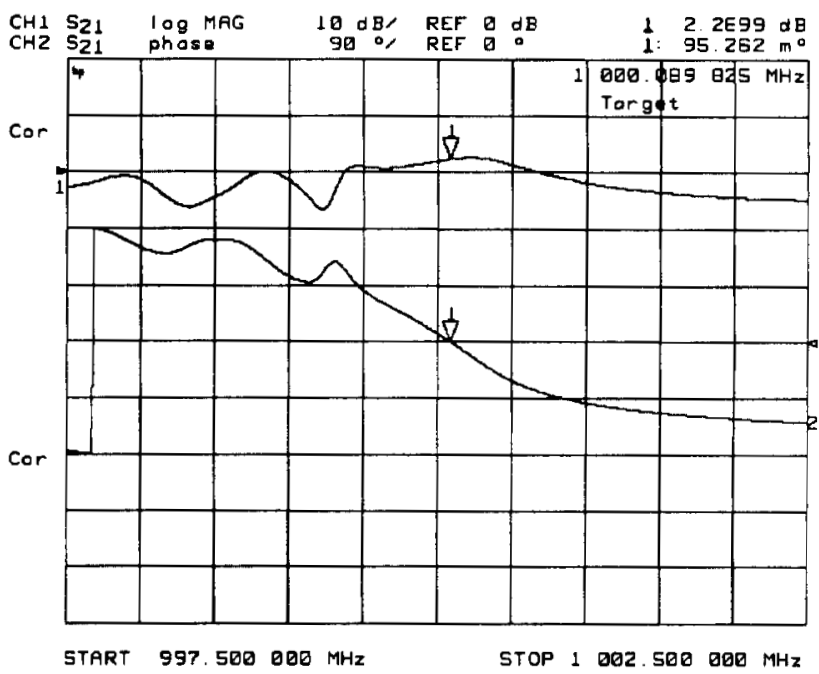

Figure 8. Open loop phase and gain. The loop is marked where the net phase change is zero. The loop gain is recorded at $2.27 \mathrm{~dB}$.

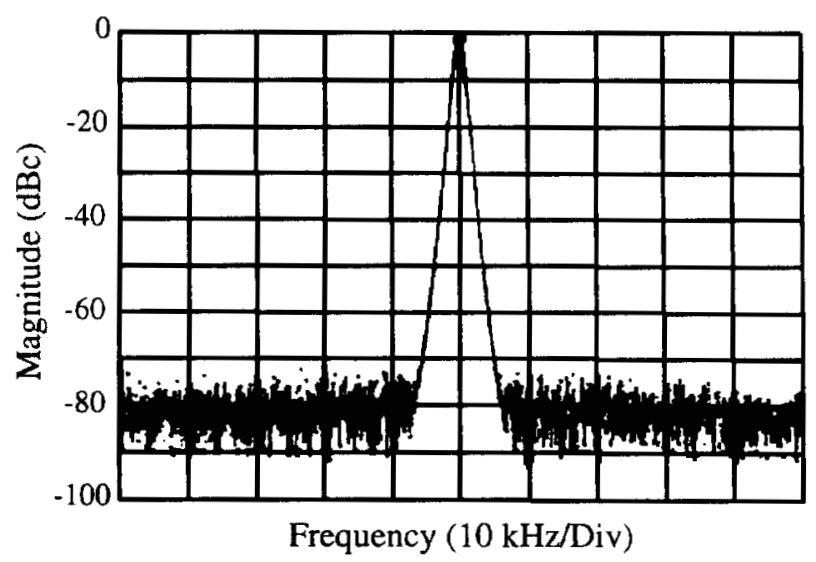

Figure 9. Output spectrum of the $1 \mathrm{GHz}$ oscillator. The center frequency is at $1.0001042 \mathrm{GHz}$. The span of the spectrum is $100 \mathrm{kHz}$. The vertical scale is $10 \mathrm{~dB} / \mathrm{Div}$.

(loop phase change of zero), the net loop gain is $2.27 \mathrm{~dB}$. Figure 9 shows the output spectrum of the oscillator around the center frequency of $1.00 \mathrm{GHz}$.

All vibration testing was done by applying a vibration of frequency $f_{v}$ in a direction normal to the propagation plane of the acoustic wave. Applying a vibration to the oscillator results in the appearance of sidebands in the output spectrum of the oscillator as seen in Fig. 1. The ratio of power in the 1st vibration induced sideband to the power at the center lobe is dependent upon the acceleration sensitivity of the oscillator. The acceleration sensitivity of the oscillator is calculated as [2]. 


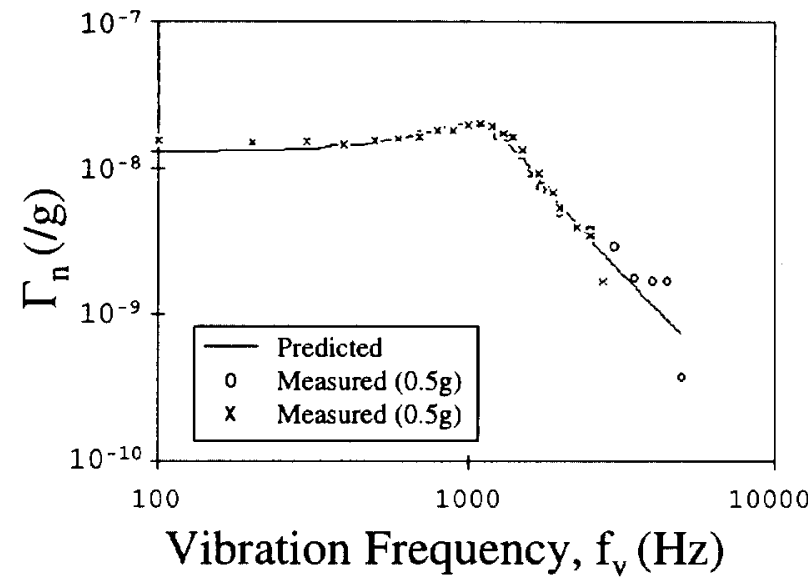

Figure 10. Measured and predicted normal vibration sensitivity of oscillator 1 . Both measurements were taken with a $0.5 \mathrm{~g}$ magnitude vibration.

$$
\Gamma_{n}=\left(\frac{2 f_{v}}{a_{n} f_{0}}\right) 10^{L_{v}(d B c) / 20}
$$

where $\Gamma_{n}$ is the normal acceleration sensitivity, $f_{v}$ is the vibration frequency, $f_{0}$ is the output frequency of the oscillator, $a_{n}$ is the magnitude of the acceleration in the normal direction, and $L_{v}(d B c)$ is the power (in Decibels) in the first vibration induced sideband relative to the power at the center frequency.

Measurements were taken from three separate oscillator units. Two of the units were mounted as shown in Fig. 6 , with a 20-25 $\mu \mathrm{m}$ separation resulting from the epoxy. The third oscillator was configured with an additional silicon spacer that increased the separation between the support platform and the oscillator substrate to $450 \mu \mathrm{m}$. These oscillators were numbered 1,2 , and 3, where oscillator 3 has the $450 \mu \mathrm{m}$ separation. After testing of oscillator 1 was completed, epoxy was placed under the support platform to rigidly mount it to the oscillator substrate. Vibration sensitivity measurements were then retaken for this oscillator. This final oscillator is referred to as the baseline oscillator.

Figure 10 shows two sets of data measured from oscillator 1 compared to the predicted output for a $20.5 \mu \mathrm{m}$ separation. Figure 11 shows the output of the baseline oscillator compared to the data from oscillator 1. Figure 12 shows the output from oscillator 2 compared with the output of the baseline oscillator. Finally, Fig. 13 shows the output of oscillator 3 compared with the output of the baseline oscillator.

As seen in Fig 10, the measured data from oscillator 1 fits the predicted curve well. The data shows a low frequency

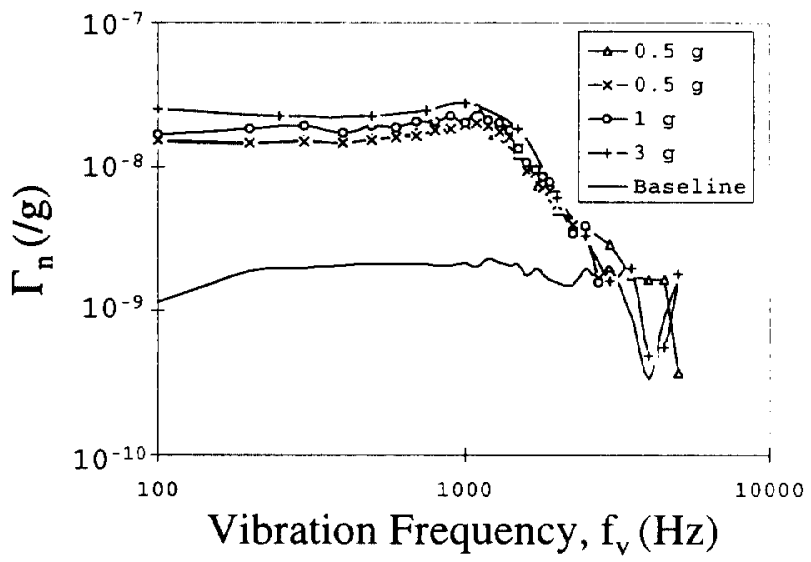

Figure 11. Measured normal vibration sensitivity of oscillator 1 and the baseline oscillator.

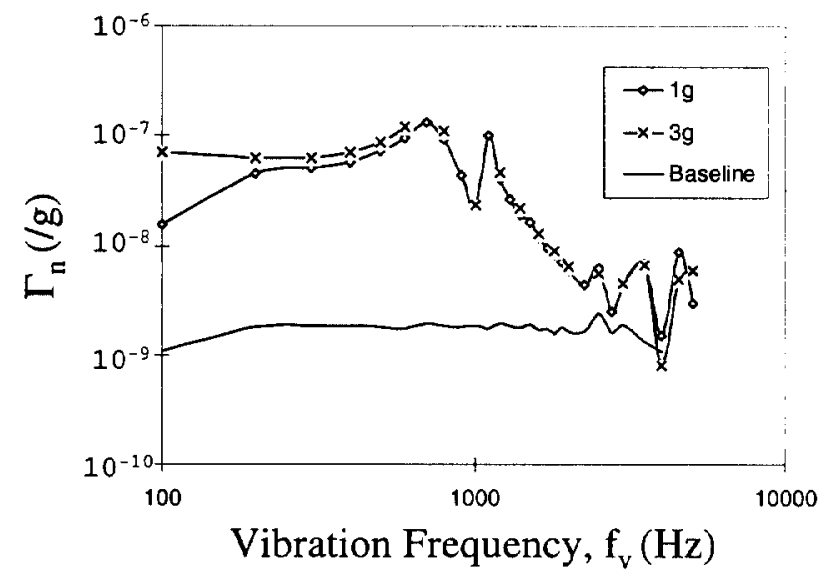

Figure 12. Measured normal vibration sensitivity of oscillator 2 and the baseline oscillator.

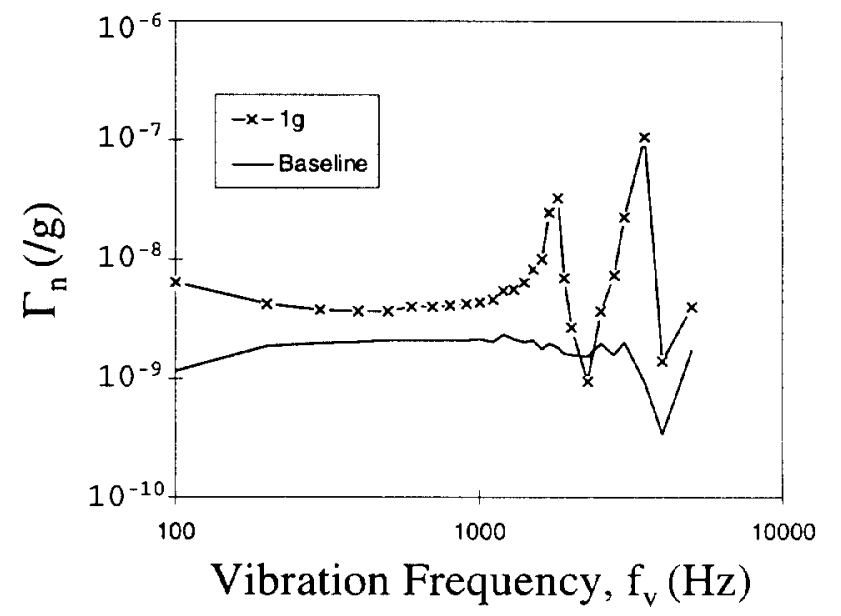

Figure 13. Measured normal vibration sensitivity of oscillator 3 and the baseline oscillator. 
vibration sensitivity of $\Gamma_{\mathrm{n}}=1.8 * 10^{-8} / \mathrm{g}$. As expected, vibration isolation is achieved for frequencies above 1.2 $\mathrm{kHz}$. The observed high frequency roll off is measured at $12 \mathrm{~dB} /$ octave. It is clear from Fig. 11 that the vibration isolation curve is a result of the isolation system since the baseline oscillator is the same as oscillator 1 except that the support platform of the baseline oscillator has been secured with epoxy. It is also apparent that the support platform is not sufficiently rigid. This is clear from the fact that the baseline oscillator has a lower vibration sensitivity in the low frequency range.

The measured values for oscillator 2 show a second peak. This peak is most likely due to a second vibrational mode of the oscillator platform. This mode may be present due to damage of the isolation platform, or from the resonator being mounted off center. However, improved process control should alleviate this problem.

The measured data for oscillator 3 also shows two resonant peaks. The large size of the peaks is a result of the decreased damping due to the increased separation between the support platform and the hybrid oscillator substrate.

\section{Discussion}

Micromachined isolation systems offer several potential advantages over other methods for reducing vibration sensitivity. In particular, the micromachined systems require a small area, have a low mass, and can be inexpensively produced. Further, the micromachined systems can be incorporated with a large variety of existing resonators and oscillators. These advantages have been demonstrated with the design of the isolation system in this research. However, two primary areas need to be addressed to enable the isolation systems to achieve state of the art performance. First, the acceleration sensitivity of the isolated STW resonators is high (1.8 ${ }^{*} 10^{-8} / \mathrm{g}$ ), and second the mechanical resonance of the system is also high $(1.1 \mathrm{kHz})$. It is important to note that the current design is a first generation system and therefore, a large variety of improvements can be incorporated to improve the system performance.

The acceleration sensitivity of the isolated resonators is two orders of magnitude worse than state of the art values reported for both SAW [19] and STW [8,9] resonator based oscillators. Reducing this value can be done by increasing the rigidity of the resonator/support platform combination, decreasing the mass of the resonator/support platform combination, or controlling the shape of the crystal deformation. The first two methods are of primary interest as they can be applied to any crystal resonator. The third method requires careful design of the resonator and the mounting structure, generally resulting in a system that is more difficult to fabricate.

Increasing the rigidity of the resonator/support platform combination can be accomplished by careful mechanical design of the support platform, or by the use of resonator support package such as a surface mount package. The use of a surface mount package will result in a significant improvement in the rigidity of the resonator, and a corresponding decrease in acceleration sensitivity. However, the package adds significant mass to the resonator/support platform structure, and thus results in increased force being applied to the support platform and the resonator for a given acceleration. A tradeoff exists between improving rigidity and increasing the supported mass. An increase in the mass leads to increased force, however, if the increased force is offset by improved rigidity, the net result is still a decrease in resonator deformation and therefore a decrease in acceleration sensitivity. Further, the increased mass results in a decreased resonant frequency allowing the vibration isolation to begin at a lower frequency. However, if there is not a sufficient increase in rigidity, the increased force results in increased deformation and acceleration sensitivity.

The second primary problem is the high mechanical resonance frequency $(1.1 \mathrm{kHz})$. In many systems, such as aircraft, the primary vibrations are in frequencies ranging from $300 \mathrm{~Hz}$ to $2 \mathrm{kHz}$. It is desirable for the vibration isolation to occur over this range of vibration frequencies. Lowering the mechanical resonance can be accomplished by decreasing the spring constant or increasing the mass. As described above, any increase in mass results in increasing acceleration sensitivity unless a corresponding improvement in rigidity is achieved. Therefore, the primary focus is on reducing the spring constant. This can be accomplished by using a polymer, such as a polyimide, to fabricate the mechanical supports. Polymers have a significantly lower Young's modulus than crystalline silicon resulting in a reduced spring constant. The design and dimensions of the supports can also be modified to reduce the spring constant further.

\section{Conclusions}

A micromachined vibration isolation system has been designed and fabricated around a commercial $1 \mathrm{GHz}$ STW resonator. The system has a small size (less than $1 \mathrm{~cm}$ by $1 \mathrm{~cm}$ by $1 \mathrm{~mm}$ ) and a low mass (less than 0.1 gram) allowing it to be utilized in applications such as missile seekers and mobile platforms where the available area and payload is limited. Further, the fabricated system demonstrates the ability to incorporate micromachined systems with existing technologies, thus reducing design 
time and cost. The micromachined systems are thus a complementary technology providing the system designer with a new technique to reduce vibration sensitivity. Also, the isolation systems can be batch fabricated, thus, allowing low cost production of the systems.

This research has shown that micromachined vibration isolation systems can be used to reduce the vibration sensitivity of crystal based oscillators. Further, areas of future research offer promise to achieve state of the art acceleration sensitivity levels combined with vibration isolation, allowing extremely low levels of oscillator vibration sensitivity. The micromachined isolation systems developed in this research offer a method to provide low cost, low mass, small size vibration isolation that can be incorporated with existing and future oscillator technology.

\section{Acknowledgments}

This research was funded by U.S. Army Research Laboratory, Physical Sciences Directorate. All finite element calculations were performed on a Cray C-90 (pk@cewes.army.mil) on time provided by the Army Corps of Engineers, CEWES station. STW resonators and hybrid oscillators were provided by SAWTEK, Inc. The authors would like to thank Dr. James T. Stewart for his assistance with the finite element analysis and the many people at SAWTEK, Inc. who supported this work.

\section{References}

[1] T.E. Parker and G.K. Montress, "Precision surfaceacoustic-wave (SAW) oscillators," IEEE Trans. on Ultrasonics, Ferroelectrics, and Frequency Control, Vol. 35, No. 3, pp. 342-364, May 1988.

[2] R.L. Filler, "The acceleration sensitivity of quartz crystal oscillators: a review," IEEE Trans. on Ultrasonics, Ferroelectrics, and Frequency Control, Vol. 35, No, 3, pp. 297-305, May 1988.

[3] J.A. Kosinski, "The fundamental nature of acceleration sensitivity," in Proc. 50th Annual Frequency Control Symp., pp. 439-448, June 1996.

[4] J.A. Kosinski and A. Ballato, "Designing for low acceleration sensitivity," IEEE Trans. on Ultrasonics, Ferroelectrics, and Frequency Control, Vol. 40, No. 5, pp. 532-537, Sept. 1993.

[5] J.A. Kosinski and A. Ballato, "Advances in acceleration sensitivity measurement and modeling," in Proc. 46th Annual Frequency Control Symp., pp. 838$848,1992$.

[6] T.E. Parker and J. Callerame, "Sensitivity of SAW delay lines and resonators to vibration," in Proc. IEEE Ultrasonics Symposium, vol. 1, pp. 129-134, 1981.
[7] B.K. Sinha and S. Locke, "Acceleration and vibration sensitivity of SAW devices," IEEE Trans. on Ultrasonics, Ferroelectrics, and Frequency Control, Vol. 34, No. 1, pp. 29-38, Jan. 1987.

[8] M.S. Cavin and R.C. Almar, "An oscillator design using low g-sensitivity, low phase noise STW devices," in Proc. 49th Annual Frequency Control Symposium, pp. 476-485, 1995.

[9] R.C. Almar and M.S. Cavin, "Low g-sensitivity fixed-frequency oscillators," Microwave Journal, pp. 8898, Feb. 1995.

[10] J.R. Reid V.M. Bright, J.T. Stewart, and J.A. Kosinski, "Reducing the normal acceleration sensitivity of surface transverse wave resonators using micromachined isolation systems," in Proc. 50th Annual Frequency Control Symposium, pp. 464-472, 1996.

[11] J.R. Reid, and V.M. Bright, "A process for the fabrication of micromechanical vibration isolation systems for reducing the vibration sensitivity of acoustic wave resonators," submitted to Sensors and Actuators A, Nov. 1996.

[12] J.T. Stewart, R. McGowan, J.A. Kosinski, and A. Ballato, "Semi-analytical finite element analysis of acceleration-induced frequency changes in SAW resonators," in Proc. 49th Annual Frequency Control Symp., pp. 499-506, 1995.

[13] J.T. Stewart, J.A. Kosinski, and A. Ballato, "An analysis of the dynamic behavior and acceleration sensitivity of a SAW resonator supported by flexible beams," in Proc. 49th Annual Frequency Control Symp., pp. 507-513, 1995.

[14] H.F. Tiersten, "Perturbation theory for linear electroelastic equations for small fields superposed on a bias," J. Acoust. Soc. Am., Vol. 64, No. 3, pp. 832-837, Sept. 1978.

[15] M. Andrews, I. Harris, and G. Turner, "A comparison of squeeze-film theory with measurements on a microstructure," Sensors and Actuators A, vol. 36, pp. 70-87, 1993.

[16] D.L. Kendall, "On etching very narrow grooves in silicon,” Appl. Phys. Letters, Vol. 26, pp. 195-198, 1975.

[17] K.E. Bean, "Anisotropic etching of silicon," IEEE Trans. Electron Devices, Vol. ED-25, pp. 1185-1192, 1978.

[18] O. Tabata, R. Asahi, H. Funabashi, K. Shimaoka, and S. Sugiyama, "Anisotropic etching of silicon in TMAH solutions," Sensors and Actuators, Vol. A34, pp. 51-57, 1992.

[19] D. Andres and T.E. Parker, "Designing smaller SAW oscillators for low vibration sensitivity," in Proc. 48th Annual Frequency Control Symp., pp. 352-358, 1994. 